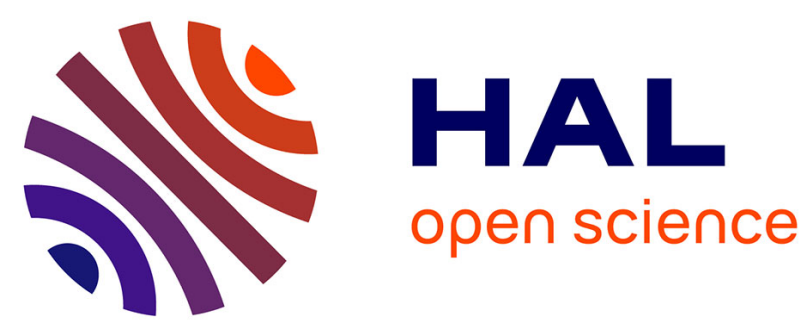

\title{
FEM-BEM coupling methods for Tokamak plasma axisymmetric free-boundary equilibrium computations in unbounded domains
}

\author{
Blaise Faugeras, Holger Heumann
}

\section{To cite this version:}

Blaise Faugeras, Holger Heumann. FEM-BEM coupling methods for Tokamak plasma axisymmetric free-boundary equilibrium computations in unbounded domains. Journal of Computational Physics, 2017, 343, pp.201 - 216. 10.1016/j.jcp.2017.04.047 . hal-01637945

\section{HAL Id: hal-01637945 https://hal.science/hal-01637945}

Submitted on 18 Nov 2017

HAL is a multi-disciplinary open access archive for the deposit and dissemination of scientific research documents, whether they are published or not. The documents may come from teaching and research institutions in France or abroad, or from public or private research centers.
L'archive ouverte pluridisciplinaire HAL, est destinée au dépôt et à la diffusion de documents scientifiques de niveau recherche, publiés ou non, émanant des établissements d'enseignement et de recherche français ou étrangers, des laboratoires publics ou privés. 


\title{
FEM-BEM coupling methods for tokamak plasma axisymmetric free-boundary equilibrium computations in unbounded domains
}

\author{
Blaise Faugeras, Holger Heumann ${ }^{1}$ \\ CASTOR Team, INRIA Sophia-Antipolis and Université de Nice \\ Parc Valrose, 06108 Nice cedex 02, FR
}

\begin{abstract}
Incorporating boundary conditions at infinity into simulations on bounded computational domains is a repeatedly occurring problem in scientific computing. The combination of finite element methods (FEM) and boundary element methods (BEM) is the obvious instrument, and we adapt here for the first time the two standard FEM-BEM coupling approaches to the free-boundary equilibrium problem: the Johnson-Nédélec coupling and the Bielak-MacCamy coupling. We recall also the classical approach for fusion applications, dubbed according to its first appearance von-Hagenow-Lackner coupling and present the less used alternative introduced by Albanese, Blum and de Barbieri in [2]. We show that the von-Hagenow-Lackner coupling suffers from undesirable non-optimal convergence properties, that suggest that other coupling schemes, in particular Johnson-Nédélec or Albanese-Blum-de Barbieri are more appropriate for non-linear equilibrium problems. Moreover, we show that any of such coupling methods requires Newton-like iteration schemes for solving the corresponding non-linear discrete algebraic systems.
\end{abstract}

\section{Introduction}

Numerical equilibrium computation is undoubtedly of first importance in Tokamak fusion science [40] and has been studied for a long time with already a review article in 1991 [38]. From a Tokamak operation point of view equilibrium codes are essential to design the geometry of new machines, to set up discharge scenarios and to check their feasibility, or to design and validate plasma feedback controllers. To this end these $2 \mathrm{D}$ equilibrium codes can also be coupled to 1D transport codes in order to simulate the evolution of the plasma equilibrium

\footnotetext{
Email addresses: blaise.faugeras@unice.fr (Blaise Faugeras),

holger.heumann@inria.fr (Holger Heumann)

${ }^{1}$ Corresponding author.
} 
at the diffusion timescale throughout the discharge [21]. More detailed magnetohydrodynamic simulations modeling the plasma on very short timescales also rely on a given initial equilibrium which is the output of these equilibrium codes. As a last example let us mention that equilibrium computation methods are also used in equilibrium reconstruction codes which aim at identifying the toroidal current density in the plasma from experimental measurements (e.g. $[31,32,7,8,33])$.

A code which treats the quasi-static free-boundary equilibrium problem needs to solve non-linear elliptic or parabolic problems with non-linear source terms representing the current density profile vanishing outside the unknown free boundary of the plasma. The computational challenges in the design of such a code are: a problem setting in an unbounded domain with a non-linearity due to the current density profile in the unknown plasma domain and the non-linear magnetic permeability if the machine has ferromagnetic structures.

In this paper we focus on how the simulation on the unbounded domain can be reduced to computations on an interior bounded domain thanks to analytical Green's functions [30]. The numerical solution on the interior domain is coupled through boundary conditions to the Green's function representation of the solution in the unbounded exterior domain. This approach is used in many other application areas such as electromagnetics $[22,41,4]$ or elasticity $[12,5,37]$ and falls in the framework of boundary integral equations. The boundary integral equations enable to reduce problems on unbounded domains to problems on boundaries which can then be coupled to any numerical method for the interior bounded domain. Most authors in the fusion literature deal with this question using a method introduced by von Hagenow and Lackner [39, 30], whereas the coupling could be also conceived in other ways. In this work, we give a succinct but self-consistent presentation of four different such schemes and discuss their performance and accuracy.

As aforementioned, certainly the most famous coupling in the fusion community is called in this paper the von Hagenow-Lackner coupling HL [39, 30]. A method implementing this coupling is present in many equilibrium codes which usually make use of a finite difference discretization method and of fixed-point iterations to solve the non-linearities. Here we propose a variational framework for this coupling which enables the use of a finite element method (FEM) combined with a boundary element method (BEM) and Newton's method for the non-linearities. Surprisingly this method does not seem to be known in the applied mathematics or scientific computing literature.

Much less known and used but nevertheless existing in the fusion literature is the analytic uncoupling on a semi-circular domain ABB introduced by Albanese, Blum and de Barbieri in [2]. It is the method implemented in the codes Proteus [3], and the more recent CREATE-NL+ [1] or CEDRES++ and FEEQS.M $[19,20]$. Such an uncoupling method was also analysed for the case of the Laplacian operator in [23] and [17].

The other two methods we will discuss in this work are very well known in the applied mathematics literature and often referred to as the Johnson-Nédélec coupling JN [42, 28, 35] and the Bielak-MacCamy coupling BMC [5]. From our 
point of view JN might be the most natural way to deal with the unbounded domain problem in the framework of a finite element method. However, neither JN nor BMC have ever been tested before in a fusion equilibrium code.

The outline of the paper is the following. In Section 2 we recall the plasma equilibrium equations in a Tokamak and present afterwards in Section 3 the boundary integral equations and the different coupling methods. Section 4 deals with the Galerkin formulations leading to the FEM-BEM discretizations of the four different coupling methods. Numerical experiments are conducted in Section 5 , where we study the approximation order, computational complexity and the difference between Newton iterations and fixed point iterations. We conclude with a short summary and outlook in Section 6 .

\section{Equilibrium equation}

We consider the magnetostatic problem

$$
\operatorname{curl}\left(\frac{1}{\mu} \operatorname{curl} \mathbf{A}\right)=\mathbf{J}
$$

of the electromagnetic vector potential $\mathbf{A}$ for some given current density $\mathbf{J}$ with $\mu$ the permeability. Under the axisymmetry assumption it is rewritten in cylindrical coordinates

$$
\begin{aligned}
-\nabla \cdot\left(\frac{1}{\mu_{0} x_{r}} \nabla \psi(\mathbf{x})\right)=\mathbf{J}(\mathbf{x}) \cdot \mathbf{e}_{\varphi} \\
\psi(\mathbf{x})_{\mid x_{r}=0}=0 ; \quad \lim _{\|\mathbf{x}\| \rightarrow+\infty} \psi(\mathbf{x})=0
\end{aligned}
$$

where $\nabla$ is the gradient in the two dimensional half space $\mathbb{H}:=[0, \infty] \times[-\infty, \infty]$ and $\mathbf{x}=\left(x_{r}, x_{z}\right)$. The primal unknown $\psi$ is the poloidal magnetic flux $\psi(\mathbf{x}):=$ $x_{r} \mathbf{A}(\mathbf{x}) \cdot \mathbf{e}_{\varphi}$, the scaled toroidal component of the vector potential $\mathbf{A}$, i.e. $\mathbf{B}=$ curl $\mathbf{A}$ and $\mathbf{e}_{\varphi}$ the unit vector in toroidal direction. We consider air transformer tokamaks only, that is to say that the permeability is the constant $\mu_{0}$ everywhere and the non-linearities are only due to the plasma domain and current density.

In the free boundary equilibrium problem considered here the toroidal component of the current density is given by

$$
\mathbf{J}(\mathbf{x}) \cdot \mathbf{e}_{\varphi}= \begin{cases}j\left(x_{r}, \psi(\mathbf{x})\right) & \text { in } \mathcal{P}(\psi), \\ j_{\mathcal{C}_{i}} & \text { in } \mathcal{C}_{i} \quad i=1, \ldots n \\ 0 & \text { elsewhere }\end{cases}
$$

with $j_{\mathcal{C}_{i}}=I_{i} /\left|\mathcal{C}_{i}\right|$ is the given constant current density in the $i$-th poloidal field coil $\mathcal{C}_{i} \subset \mathbb{H}$ and $j\left(x_{r}, \psi(\mathbf{x})\right)$ the prescribed toroidal component of the plasma current density, generally a non-linear function of $\psi$, in the plasma domain $\mathcal{P}(\psi) \subset \mathcal{L} \subset \mathbb{H}$ with $\mathcal{L}$ the limiter-bounded domain that is accessible to the plasma. The plasma domain $\mathcal{P}(\psi)$ is the domain bounded by the last closed 


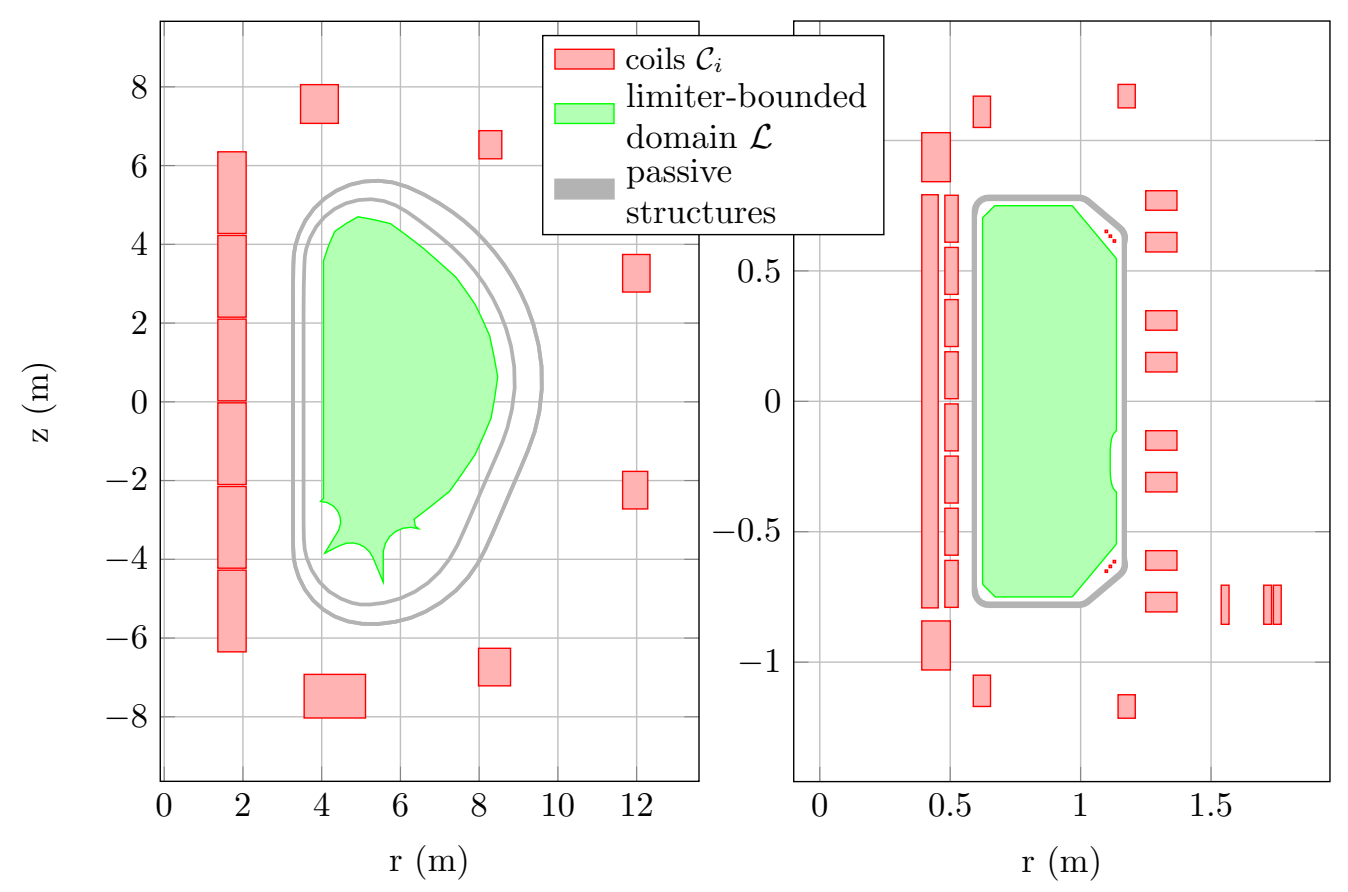

Figure 1: The poloidal section of the Tokamaks ITER (left) and TCV (right).

poloidal flux line inside the limiter bounded domain $\mathcal{L}$. Figure 1 shows the sections of two Tokamaks that illustrate the geometric setting of the free-boundary equilibrium problem (1) and in (28) we provide an example for $j\left(x_{r}, \psi(\mathbf{x})\right)$.

Hence, the axisymmetric magnetostatic problem is a non-linear problem, which, due to the unknown plasma domain $\mathcal{P}(\psi)$ is called the free-boundary equilibrium problem. We refer to standard text books (e.g. [16, 6, 40, 18, 27]) for further details on the derivation of this model.

\section{Boundary integral coupling methods}

To solve problem (1) numerically we need to find a reformulation on a bounded domain $\Omega$, the computational domain, containing the plasma domain $\mathcal{P}(\psi)$. One is tempted to choose $\Omega$ as small as possible. But the equilibrium problem (1) is non-linear in the limiter-bounded domain $\mathcal{L}$ due to the righthand side and the unknown plasma domain $\mathcal{P}$, and hence, the computational domain $\Omega$ should contain at least $\mathcal{L}$. It might be even convenient to include in $\Omega$ also some or all coils (e.g. the small coils next to the limiter in TCV, Figure 1) and the passive structures. Moreover the shape of the limiter can be irregular (e.g. for ITER, Figure 1), which impedes to use directly the limiter as the interface of FEM-BEM coupling. Therefore, to stay as general as possible, we are not assuming that the computational domain $\Omega$ contains either all or no coils. We 
introduce the two index subsets $I=\left\{i / \mathcal{C}_{i} \subset \Omega\right\}$ and $I^{\mathrm{c}}=\left\{i / \mathcal{C}_{i} \subset \mathbb{H} \backslash \Omega\right\}$ to distinguish coils contained in the computational domain $\Omega$ and coils contained in the complement $\mathbb{H} \backslash \Omega$.

The coupling of the solution in the computational domain $\Omega$ with the solution on the complement $\mathbb{H} \backslash \Omega$ is ensured by appropriate non-local boundary conditions. The boundary conditions are given by boundary integral equations that follow from Green's identities for the differential operator $\Delta^{*}:=\nabla \cdot\left(\frac{1}{\mu_{0} x_{r}} \nabla \cdot\right)$, which we recall here for an arbitrary domain $D \subset \mathbb{H}$.

Boundary integral equations. Namely, for any domain $D \subset \mathbb{H}$, with piecewise differentiable boundary and all regular enough $\phi$ and $\xi$ it holds that (see e.g. [36, page $1-3$, eq. 1.8$]$ or $[25$, page 428$])$

$$
\begin{aligned}
& \int_{D}\left(\phi(\mathbf{y}) \Delta^{*} \xi(\mathbf{y})-\xi(\mathbf{y}) \Delta^{*} \phi(\mathbf{y})\right) d \mathbf{y}+ \\
& \int_{\partial D}\left(\partial_{\mathbf{n}(\mathbf{y})}^{*} \phi(\mathbf{y}) \xi(\mathbf{y})-\partial_{\mathbf{n}(\mathbf{y})}^{*} \xi(\mathbf{y}) \phi(\mathbf{y})\right) d s(\mathbf{y})=0,
\end{aligned}
$$

where $\mathbf{y}=\left(y_{r}, y_{z}\right), \mathbf{n}$ is the outward normal vector on $\partial D$ and $\partial_{\mathbf{n}}^{*} \xi(\mathbf{x})=$ $\frac{1}{\mu_{0} x_{r}} \nabla \xi(\mathbf{x}) \cdot \mathbf{n}$.

Let us also introduce the fundamental solution of $-\Delta^{*}[26]$ which writes explicitly as

$$
G(\mathbf{x}, \mathbf{y})=\frac{\mu_{0} \sqrt{x_{r} y_{r}}}{2 \pi k(\mathbf{x}, \mathbf{y})}\left(\left(2-k^{2}(\mathbf{x}, \mathbf{y})\right) K(k(\mathbf{x}, \mathbf{y}))-2 E(k(\mathbf{x}, \mathbf{y}))\right),
$$

with

$$
k^{2}(\mathbf{x}, \mathbf{y})=\frac{4 x_{r} y_{r}}{\left(x_{r}+y_{r}\right)^{2}+\left(x_{z}-y_{z}\right)^{2}},
$$

and $K(k)$ and $E(k)$ are the complete elliptic integrals of the first and second kind respectively. Hence, taking in $(3) \phi(\mathbf{y})=G(\mathbf{x}, \mathbf{y})$ we have the integral identity (see e.g. [36, page 89, eq. 5.2] or [25, eq. 9]) in $D$ :

$$
\begin{aligned}
\xi(\mathbf{x})+\int_{\partial D} \partial_{\mathbf{n}(\mathbf{y})}^{*} G(\mathbf{x}, \mathbf{y}) \xi(\mathbf{y}) d s(\mathbf{y})- & \int_{\partial D} \partial_{\mathbf{n}(\mathbf{y})}^{*} \xi(\mathbf{y}) G(\mathbf{x}, \mathbf{y}) d s(\mathbf{y}) \\
& =-\int_{D} G(\mathbf{x}, \mathbf{y}) \Delta^{*} \xi(\mathbf{y}) d \mathbf{y} \quad \forall \mathbf{x} \in D
\end{aligned}
$$

for all regular enough $\xi$. Further, it can be shown (see e.g. [36, page 137, eq. 6.20 ,] or $[25$, eq. 11]) that in the limit $\mathbf{x} \in \partial D$ the following integral identity holds:

$$
\begin{aligned}
\frac{1}{2} \xi(\mathbf{x})+\int_{\partial D} \partial_{\mathbf{n}(\mathbf{y})}^{*} G(\mathbf{x}, \mathbf{y}) \xi(\mathbf{y}) d s(\mathbf{y}) & -\int_{\partial D} \partial_{\mathbf{n}(\mathbf{y})}^{*} \xi(\mathbf{y}) G(\mathbf{x}, \mathbf{y}) d s(\mathbf{y}) \\
& =-\int_{D} G(\mathbf{x}, \mathbf{y}) \Delta^{*} \xi(\mathbf{y}) d \mathbf{y} \quad \mathbf{x} \in \partial D
\end{aligned}
$$


von Hagenow-Lackner coupling HL $[\mathbf{3 9}, \mathbf{3 0}]$. Green's second identity (3) for $D=\mathbb{H}$ with $\phi=G$ and $\xi=\psi$ the solution of (1), leads to a non-linear integral equation for $\psi$ :

$$
\psi(\mathbf{x})=\int_{\mathcal{P}(\psi)} j\left(y_{r}, \psi(\mathbf{y})\right) G(\mathbf{x}, \mathbf{y}) d \mathbf{y}+\sum_{i \in I \cup I^{\mathrm{c}}} \int_{\mathcal{C}_{i}} j_{\mathcal{C}_{i}} G(\mathbf{x}, \mathbf{y}) d \mathbf{y} \quad \forall \mathbf{x} \in \mathbb{H} .
$$

In particular this provides a formula for the Dirichlet conditions of $\psi$ on the boundary $\partial \Omega$ of the computational domain $\Omega$. Hence it is possible to reformulate the free-boundary equilibrium problem in the unbounded domain (1) as a Dirichlet boundary value problem in the bounded domain $\Omega$ using expression (6) as the Dirichlet boundary condition.

In order to avoid the computation of the integral over the possible large domain $\mathcal{P}(\psi)$ when evaluating (6), one then introduces a new auxiliary unknown $u$ satisfying the homogeneous Dirichlet boundary value problem

$$
-\Delta^{*} u(\mathbf{x})=j\left(x_{r}, \psi\right) \chi_{\mathcal{P}(\psi)}(\mathbf{x})+\sum_{i \in I} j_{\mathcal{C}_{i}} \chi_{\mathcal{C}_{i}}(\mathbf{x}) \quad \text { in } \Omega, \quad u=0 \quad \text { on } \partial \Omega,
$$

where $\chi$ is the domain indicator function. Green's third identity (5) for $D=\Omega$ with $\xi=u$ leads to

$$
\begin{aligned}
\int_{\mathcal{P}(\psi)} j\left(y_{r}, \psi(\mathbf{y})\right) G(\mathbf{x}, \mathbf{y}) d \mathbf{y}+ & \sum_{i \in I} \int_{\mathcal{C}_{i}} j_{\mathcal{C}_{i}} G(\mathbf{x}, \mathbf{y}) d \mathbf{y} \\
& =\int_{\partial \Omega} \partial_{\mathbf{n}(\mathbf{y})}^{*} u(\mathbf{y}) G(\mathbf{x}, \mathbf{y}) d s(\mathbf{y}) \quad \forall \mathbf{x} \in \partial \Omega,
\end{aligned}
$$

with $\mathbf{n}(\mathbf{y})$ the inward pointing normal of $\Omega$, showing that the integral over plasma domain and coils in $\Omega$ in equation (6) can be replaced by an integral over the boundary $\partial \Omega$ using the Neumann data of $u$, the solution to problem (7). Hence the Dirichlet boundary condition on $\partial \Omega$ is expressed as the sum of the boundary integral in (8) involving the new unknown $u$ and the Green function convolutions term of the currents flowing in $\mathbb{H} \backslash \Omega$.

Johnson-Nédélec coupling JN, direct method [42, 28]. One introduces a supplementary unknown $q \approx \partial_{\mathbf{n}}^{*} \psi$ for the Neumann data on $\partial \Omega$, where $\mathbf{n}$ is the inward pointing normal of $\Omega$. Green's third identity (5) for $\xi=\psi$ and $D=\mathbb{H} \backslash \Omega$ gives a supplementary boundary integral equation:

$$
\begin{aligned}
& \frac{1}{2} \psi(\mathbf{x})+\int_{\partial \Omega}\left(\partial_{\mathbf{n}(\mathbf{y})}^{*} G(\mathbf{x}, \mathbf{y}) \psi(\mathbf{y})-q(\mathbf{y}) G(\mathbf{x}, \mathbf{y})\right) d s(\mathbf{y}) \\
& =\sum_{i \in I^{\mathrm{c}}} \int_{\mathcal{C}_{i}} j_{\mathcal{C}_{i}} G(\mathbf{x}, \mathbf{y}) d \mathbf{y} \quad \forall \mathbf{x} \in \partial \Omega .
\end{aligned}
$$

So, JN amounts to couple a Neumann data boundary value problem for $\psi$ in $\Omega$ with the integral equation (9) that involves as well $\psi$ and the Neumann data $q$. 
Bielak-MacCamy coupling BMC, indirect method [5]. One introduces a supplementary unknown potential $q$ on $\partial \Omega$, and defines an auxiliary unknown $\chi(\mathbf{x})$ for $\mathbf{x} \in \mathbb{H} \backslash \Omega$, based on a boundary integral over the potential $q$

$$
\chi(\mathbf{x}):=\int_{\partial \Omega} G(\mathbf{x}, \mathbf{y}) q(\mathbf{y}) d s(\mathbf{y})+\sum_{i \in I^{\mathrm{c}}} \int_{\mathcal{C}_{i}} j_{\mathcal{C}_{i}} G(\mathbf{x}, \mathbf{y}) d \mathbf{y},
$$

and finds, again by Green's theorem, that

$$
-\Delta^{*} \chi(\mathbf{x})=\sum_{i \in I^{\mathrm{c}}} \int_{\mathcal{C}_{i}} j_{\mathcal{C}_{i}} G(\mathbf{x}, \mathbf{y}) d \mathbf{y} \quad \text { in } \mathbb{H} \backslash \Omega,
$$

meaning that $\chi(\mathbf{x})$ is a representation of the solution $\psi(\mathbf{x})$ of $(1)$ when $\mathbf{x} \in \mathbb{H} \backslash \Omega$.

In the limit cases $\mathbf{x} \in \partial \Omega$ we get integral representation formulas for the Dirichlet data of $\chi$

$$
\xi(\mathbf{x})=\int_{\partial \Omega} G(\mathbf{x}, \mathbf{y}) q(\mathbf{y}) d s(\mathbf{y})+\sum_{i \in I^{\mathrm{c}}} \int_{\mathcal{C}_{i}} j_{\mathcal{C}_{i}} G(\mathbf{x}, \mathbf{y}) d \mathbf{y}
$$

and the Neumann data of $\chi$

$$
\begin{aligned}
\partial_{\mathbf{n}}^{*} \chi(\mathbf{x})=\frac{1}{2} q(\mathbf{x})+\int_{\partial \Omega} \partial_{\mathbf{n}(\mathbf{x})}^{*} G(\mathbf{x}, \mathbf{y}) q(\mathbf{y}) d s(\mathbf{y}) & \\
& +\sum_{i \in I^{\mathrm{c}}} \int_{\mathcal{C}_{i}} j_{\mathcal{C}_{i}} \partial_{\mathbf{n}(\mathbf{x})}^{*} G(\mathbf{x}, \mathbf{y}) d \mathbf{y} \quad \mathbf{x} \in \partial \Omega,
\end{aligned}
$$

which are forced to be equal to the Dirichlet and Neumann data of $\psi$. Here again $\mathbf{n}$ is the inward pointing normal of $\Omega$. Hence, BMC amounts to combine the Neumann data boundary value problem for $\psi$ in $\Omega$, based on $q$-parametrized Neumann data given by the right hand side of (12), with the integral equation (11) that involves as well $\psi$ (through its Dirichlet data) and the potential $q$.

Analytic uncoupling on a semi-circular domain ABB [2] [17]. This coupling method requires a very particular shape of the computational domain. We choose $\Omega$ to be a semi-circular domain containing the limiter-bounded domain $\mathcal{L}$ and all the coils $\mathcal{C}_{i}$. Its boundary is $\partial \Omega=\Gamma \cup \Gamma_{0}$ where $\Gamma$ is the semi-circle of radius $\rho_{\Gamma}$ and $\Gamma_{0}=\left\{\mathbf{x} / x_{r}=0,-\rho_{\Gamma} \leq x_{z} \leq \rho_{\Gamma}\right\}$. This particular choice enables to find analytically, thanks to the method of images, a special Green function $G^{*}(\mathbf{x}, \mathbf{y})$ which vanishes on the semi-circle $\Gamma$. Then using Green's theorem (3) with $D=\mathbb{H} \backslash \Omega$ and $\phi=G^{*}$ one obtains

$$
\psi(\mathbf{x})=\int_{\Gamma} \psi(\mathbf{y}) \partial_{\mathbf{n}(\mathbf{y})}^{*} G^{*}(\mathbf{x}, \mathbf{y}) d s(\mathbf{y}) \quad \forall \mathbf{x} \in \Gamma .
$$

The normal derivative $\partial_{\mathbf{n}}^{*} \psi(\mathbf{x})$ can then also be computed analytically as a boundary integral depending on $\psi$ and reinjected in the boundary condition term of the weak formulation for the inner problem on $\Omega$. We refer to [19] for a detailed exposition of this approach. 


\section{Galerkin formulation}

In most of the computational tools for computing axisymmetric plasma equilibria, the finite difference method for the strong formulation (1) of the equilibrium problem is combined with HL. We follow here the more general Galerkin method, and recall that for appropriately chosen triangulations the Galerkin method leads to the same stencils as the finite difference approach. Moreover the Galerkin method allows more flexibility for approximating the realistic geometry of a Tokamak.

We consider problem (1) restricted to the bounded computational domain $\Omega$, multiply by a test function $\xi$ and do integration by parts:

$$
\int_{\Omega} \frac{1}{\mu_{0} x_{r}} \nabla \psi(\mathbf{x}) \cdot \nabla \xi(\mathbf{x}) d \mathbf{x}+\int_{\partial \Omega} \partial_{\mathbf{n}}^{*} \psi(\mathbf{x}) \xi(\mathbf{x}) d s(\mathbf{x})=\int_{\Omega} \mathbf{J}(\mathbf{x}) \cdot \mathbf{e}_{\varphi} \xi(\mathbf{x}) d \mathbf{x}
$$

where $\mathbf{n}$ is the inward pointing normal.

We use a triangular mesh to cover the computational domain $\Omega$ and introduce a basis of piecewise linear functions $\left\{\lambda_{i}\right\}$, where each $\lambda_{i}$ vanishes at all mesh vertices except one. Basis functions associated to vertices at $x_{r}=0$ are excluded from this finite element space $X(\Omega)$, as, due to axisymmetry $\psi(\mathbf{x})_{\mid x_{r}=0}=0$. The finite element space $X(\Omega)$, is the linear Lagrangian finite element space and has the direct decomposition $X(\Omega)=X_{\circ}(\Omega) \oplus X_{\partial}(\Omega)$, where $X_{\circ}(\Omega)$ is the space of all finite element functions in $X(\Omega)$ that have zero Dirichlet data. The degrees of freedom of elements of $X_{\circ}(\Omega)$ are the values at the vertices of the mesh, that are not on the boundary $\partial \Omega$ and the degrees of freedom of elements of $X_{\partial}(\Omega)$ are the values at the vertices on the boundary $\partial \Omega$. Additionally we will make use of the finite element space $Q(\Omega)$ being the span of piecewise constant functions $\left\{\chi_{i}\right\}$, where each $\chi_{i}$ vanishes everywhere except for one edge of the boundary $\partial \Omega$.

To define the different Galerkin formulations of HL, JN, BMC and ABB let us introduce the following notations for operators related to the Galerkin method on $\Omega$ :

$$
\begin{aligned}
a(\psi, \xi) & :=\int_{\Omega} \frac{1}{\mu_{0} x_{r}} \nabla \psi(\mathbf{x}) \cdot \nabla \xi(\mathbf{x}) d \mathbf{x}, \\
j_{p}(\psi, \xi) & :=\int_{\mathcal{P}(\psi)} j\left(x_{r}, \psi(\mathbf{x})\right) \xi(\mathbf{x}) d \mathbf{x}
\end{aligned}
$$

and

$$
\ell(\xi):=\sum_{i \in I} j_{\mathcal{C}_{i}} \int_{\mathcal{C}_{i}} \xi(\mathbf{x}) d \mathbf{x} .
$$

The implementation of these operators relies on quadrature rules for integrals over the triangular elements of the mesh. The approximation of the non-linear $j_{p}(\psi, \xi)$ is non-standard due to the integration domain depending on $\psi$ and details can be found in [20]. 
Moreover we will make also use of boundary integral operators and introduce

$$
\begin{array}{rlrl}
V(q)(\mathbf{x}) & :=\int_{\partial \Omega} G(\mathbf{x}, \mathbf{y}) q(\mathbf{y}) d s(\mathbf{y}), & \mathbf{x} \in \partial \Omega \\
K(\psi)(\mathbf{x}) & :=\int_{\partial \Omega} \partial_{\mathbf{n}(\mathbf{y})}^{*} G(\mathbf{x}, \mathbf{y}) \psi(\mathbf{y}) d s(\mathbf{y}), & \mathbf{x} \in \partial \Omega, \\
K^{\prime}(\psi)(\mathbf{x}):=\int_{\partial \Omega} \partial_{\mathbf{n}(\mathbf{x})}^{*} G(\mathbf{x}, \mathbf{y}) \psi(\mathbf{y}) d s(\mathbf{y}), & \mathbf{x} \in \partial \Omega,
\end{array}
$$

and domain integral operators

$$
\begin{aligned}
L(\mathbf{x}):=\sum_{i \in I^{\mathrm{c}}} j_{\mathcal{C}_{i}} \int_{\mathcal{C}_{i}} G(\mathbf{x}, \mathbf{y}) d \mathbf{y}, & \mathbf{x} \in \Omega, \\
L^{\prime}(\mathbf{x})=\sum_{i \in I^{\mathrm{c}}} j_{\mathcal{C}_{i}} \int_{\mathcal{C}_{i}} \partial_{\mathbf{n}(\mathbf{x})}^{*} G(\mathbf{x}, \mathbf{y}) d \mathbf{y}, & \mathbf{x} \in \Omega .
\end{aligned}
$$

In the subsequent Galerkin formulations we will frequently integrate products of integral operators and test functions over the boundary, hence it is convenient to introduce also

$$
\langle\psi, \xi\rangle_{\partial \Omega}:=\int_{\partial \Omega} \psi(\mathbf{x}) \xi(\mathbf{x}) d s(\mathbf{x}) .
$$

In the case where $\psi$ is one of the boundary integral operator in (17) the approximation of such inner products is non-trivial and goes beyond the standard quadrature formulas. Nevertheless, this task is well understood, and we refer to [11] for the technical details recalling also the asymptotic formulas for the fundamental solution $G(\mathbf{x}, \mathbf{y})$ when $\|\mathbf{x}-\mathbf{y}\| \rightarrow 0$ derived in [24].

Here and in what follows, we will use the symbol $\Omega^{\mathbf{D}}$ to distinguish the computational domain that verifies the assumptions for ABB from the more general computational domain $\Omega$. While $\Omega^{\boldsymbol{\top}}$ is a semi-circular domain containing the limiter-bounded domain $\mathcal{L}$ and all the coils $\mathcal{C}_{i}$, the domain $\Omega$ only requires to contain $\mathcal{L}$. In particular it is not required that $\Omega$ is a connected domain.

HL with $\Omega$. Dirichlet boundary conditions $g$ are imposed in (14) and computed using equations (6), (7) and (8). This leads to the introduction of the following Galerkin formulation: find $(\psi, g, u) \in X_{\circ}(\Omega) \times X_{\partial}(\Omega) \times X_{\circ}(\Omega)$, such that

$$
\begin{aligned}
a(\psi, \xi)+a(g, \xi)-j_{p}(\psi, \xi) & =\ell(\xi), & & \forall \xi \in X_{\circ}(\Omega), \\
\langle g, f\rangle_{\partial \Omega}-\left\langle V\left(\partial_{\mathbf{n}}^{*} u\right), f\right\rangle_{\partial \Omega} & =\langle L, f\rangle_{\partial \Omega}, & & \forall f \in X_{\partial}(\Omega), \\
a(u, v)-j_{p}(\psi, v) & =\ell(v), & & \forall v \in X_{\circ}(\Omega) .
\end{aligned}
$$

JN with $\Omega$. We supplement equation (14) for $\psi$ on $\Omega$ with boundary integral equation (9) for $q$, the auxiliary variable for the Neumann data, and obtain the following Galerkin formulation: find $(\psi, q) \in X(\Omega) \times Q(\Omega)$, such that

$$
\begin{array}{rlrl}
a(\psi, \xi)-j_{p}(\psi, \xi)+\langle q, \xi\rangle_{\partial \Omega} & =\ell(\xi), & & \forall \xi \in X(\Omega), \\
\left\langle\frac{1}{2} \psi+K(\psi), p\right\rangle_{\partial \Omega}-\langle V(q), p\rangle_{\partial \Omega} & =\langle L, p\rangle_{\partial \Omega}, & \forall p \in Q(\Omega) .
\end{array}
$$


BMC with $\Omega$. We supplement equation (14) for $\psi$ on $\Omega$ with boundary integral equation (11), use (12) for the Neumann data and obtain the following Galerkin formulation: find $(\psi, q) \in X(\Omega) \times Q(\Omega)$, such that

$$
\begin{aligned}
a(\psi, \xi)-j_{p}(\psi, \xi)+\left\langle\frac{1}{2} q+K^{\prime}(q), \xi\right\rangle_{\partial \Omega} & =\ell(\xi)-\left\langle L^{\prime}, \xi\right\rangle_{\partial \Omega}, & & \forall \xi \in X(\Omega), \\
\langle\psi, p\rangle_{\partial \Omega}-\langle V(q), p\rangle_{\partial \Omega} & =\langle L, p\rangle_{\partial \Omega}, & & \forall p \in Q(\Omega) .
\end{aligned}
$$

ABB with $\Omega^{\top}$. The Galerkin formulation for this method is given in $[6,20]$. We briefly recall it here for completeness: Find $\psi \in X\left(\Omega^{\mathbf{D}}\right)$ such that

$$
a(\psi, \xi)-j_{p}(\psi, \xi)+c(\psi, \xi)=\ell(\xi) \quad \forall \xi \in X\left(\Omega^{\mathbf{D}}\right) .
$$

The bilinear form $c(\cdot, \cdot)$ derives from (13) as detailed in [19]. It is defined as follows

$$
\begin{aligned}
c(\psi, \xi):= & \frac{1}{\mu_{0}} \int_{\Gamma} \psi(\mathbf{x}) N(\mathbf{x}) \xi(\mathbf{x}) d s(\mathbf{x}) \\
& +\frac{1}{2 \mu_{0}} \int_{\Gamma} \int_{\Gamma}(\psi(\mathbf{x})-\psi(\mathbf{y})) M(\mathbf{x}, \mathbf{y})(\xi(\mathbf{x})-\xi(\mathbf{y})) d s(\mathbf{x}) d s(\mathbf{y}),
\end{aligned}
$$

with

$$
\begin{aligned}
M(\mathbf{x}, \mathbf{y}) & =\frac{k(\mathbf{x}, \mathbf{y})}{2 \pi\left(x_{r} y_{r}\right)^{\frac{3}{2}}}\left(\frac{2-k(\mathbf{x}, \mathbf{y})^{2}}{2-2 k(\mathbf{x}, \mathbf{y})^{2}} E(k(\mathbf{x}, \mathbf{y}))-K(k(\mathbf{x}, \mathbf{y}))\right) \\
N(\mathbf{x}) & =\frac{1}{x_{r}}\left(\frac{1}{\delta_{+}}+\frac{1}{\delta_{-}}-\frac{1}{\rho_{\Gamma}}\right) \text { and } \delta_{ \pm}=\sqrt{x_{r}^{2}+\left(\rho_{\Gamma} \pm x_{z}\right)^{2}}
\end{aligned}
$$

where $\rho_{\Gamma}$ is the radius of the circle defining $\Omega^{\mathbf{D}}$.

Each of the four Galerkin formulations corresponds to a finite dimensional non-linear system $\mathbf{F}(\mathbf{U})=0$, where we provide the different dimensions in Table 1. In general we can say that $N_{\circ}=\operatorname{dim}\left(X_{\circ}(\Omega)\right)$, the number of vertices not on the boundary, is orders of magnitude larger than $N_{\partial}=\operatorname{dim}\left(X_{\partial}(\Omega)\right)$ the number of vertices on the boundary and $N_{\text {edges }}=\operatorname{dim}(Q(\Omega))$ the number of edges on the boundary. Hence, in summary the non-linear algebraic system for HL will be roughly twice as large as the non-linear algebraic system for JN and BMC. Moreover, comparing $\mathrm{HL}$, JN and $\mathrm{BMC}$ with $\mathrm{ABB}$, the requirement of $\mathrm{ABB}$ of $\Omega^{\top}$ to be a half circle seems to lead to an undesirable increase of unknowns for ABB.

On the other hand, the ultimate performance of all the four methods is only indirectly linked to the dimension. Due to the non-linearity, we need to employ iteration schemes, and so the performance is more linked to the number of iterations needed to achieve convergence and also to the computational time that is required to update from iteration $n$ to iteration $n+1$.

To keep the number of iterations small Newton type methods with their fast superlinear or even quadratic convergence are highly recommended. Newton type methods for $\mathrm{ABB}$ are advocated in the numerous contributions, starting 
with [9], since the early eighties. Without any additional technicality it is also possible to use Newton's method for the other three different formulations. The only non-trivial term in the derivative of each $\mathbf{F}$, corresponds to the derivative of $j_{p}(\psi, \xi)$, that can be found in [20], where it was introduced for the coupling approach ABB. All the codes that implement HL so far are using Picard type iterations that avoid the derivative of $j_{p}(\psi, \xi)$. The original approach [30] reads as: Given $\left(\psi^{n}, g^{n}\right) \in X_{\circ}(\Omega) \times X_{\partial}(\Omega)$ find $\left(\psi^{n+1}, g^{n+1}, u^{n+1}\right) \in X_{\circ}(\Omega) \times X_{\partial}(\Omega) \times$ $X_{\circ}(\Omega)$ such that

$$
\begin{aligned}
a\left(\psi^{n+1}, \xi\right)+a\left(g^{n}, \xi\right)-j_{p}\left(\psi^{n}, \xi\right) & =\ell(\xi), & & \forall \xi \in X_{\circ}(\Omega), \\
\left\langle g^{n+1}, f\right\rangle_{\partial \Omega}-\left\langle V\left(\partial_{\mathbf{n}}^{*} u^{n+1}\right), f\right\rangle_{\partial \Omega} & =\langle L, f\rangle_{\partial \Omega}, & & \forall f \in X_{\partial}(\Omega), \\
a\left(u^{n+1}, v\right)-j_{p}\left(\psi^{n+1}, v\right) & =\ell(v), & & \forall v \in X_{\circ}(\Omega),
\end{aligned}
$$

which has the advantage that one needs to solve in each iteration only two Dirichlet problems for the linear operator $-\Delta^{*}$. It is possible to derive highly efficient algorithms for this task combining finite differences and fast Fourier transform. Nevertheless, it is reported that such iteration schemes suffer from serious convergence problems [30, 27] and in [6] it was shown that Picard type iterations for $\mathrm{ABB}$ can lead to non-converging schemes.

In efficient implementations of either Newton or Picard type schemes for $\mathrm{HL}, \mathrm{JN}, \mathrm{BMC}$ or ABB the most time consuming part of each update will be the inversion of large linear systems. Here it is a priori not clear whether a Newton type scheme for JN and BMC is superior to a Newton type scheme for ABB: the linear systems of JN and BMC are considerably smaller than the linear systems for $\mathrm{ABB}$, but the integral equations in JN and BMC lead to dense entries in the linear system, which can demand large resources for the inversion.

Newton-type iterations are known to converge super-linearly, once the iterate is sufficiently close to the solution. But as it is not easy to quantify "sufficiently close", one generally needs to invoke so called globalization strategies. For the moment, we exclude such globalization strategies from our discussions, but assume that we have a sufficiently good initial guess. This is indeed the case in many applications, e.g. equilibrium reconstructions, where the equilibrium at the previous timestep is a good initial guess, or scenario development, where the formulation of inverse problems allows to find coil currents that correspond to a prescribed equilibrium.

\section{Numerical experiments}

All the subsequent simulations and numerical experiments were performed on a MacBook Pro with the 2,8 GHz Intel Core i7 processor and $16 \mathrm{~GB} 1600 \mathrm{MHz}$ DDR3 memory. The implementation is basically an extension of FEEQS.M ${ }^{2}$, which is a MATLAB implementation of the methods for axisymmetric free boundary plasma equilibria that are described in [20]. Concerning the details

\footnotetext{
${ }^{2}$ http://www-sop.inria.fr/members/Holger.Heumann/Software.html
} 


\begin{tabular}{c|c|c} 
method & dimension & leading order \\
\hline HL & $2 N_{\circ}+N_{\partial}$ & $O\left(2 N_{\circ}\right)$ \\
JN & $N_{\circ}+N_{\partial}+N_{\text {edges }}$ & $O\left(N_{\circ}\right)$ \\
BMC & $N_{\circ}+N_{\partial}+N_{\text {edges }}$ & $O\left(N_{\circ}\right)$ \\
ABB & $N_{\circ}+N_{\partial}$ & $O\left(N_{\circ}\right)$
\end{tabular}

Table 1: The dimensions of the finite dimensional non-linear system $\mathbf{F}(\mathbf{U})=0$ for the four different methods. $N_{\circ}=\operatorname{dim}\left(X_{\circ}(\Omega)\right)$ and $N_{\circ}^{\mathbf{O}}=\operatorname{dim}\left(X_{\circ}\left(\Omega^{\mathbf{O}}\right)\right)$ is the number of vertices not on the boundary, $N_{\partial}=\operatorname{dim}\left(X_{\partial}(\Omega)\right)$ and $N_{\partial}^{\mathbf{D}}=\operatorname{dim}\left(X_{\partial}\left(\Omega^{\top}\right)\right)$ is the number of vertices on the boundary and $N_{\text {edges }}=\operatorname{dim}(Q)$ is the number of edges on the boundary. We use the superscript to recall that $\mathrm{ABB}$ requires the computational domain to be a half circle $\Omega^{\top}$. In general $N_{\circ} \gg N_{\partial}$.

of the implementation, e.g. quadrature rules and the accurate linearizations of various terms in the Galerkin formulations (20), (21), (22) and (23), we refer to [20] and [11]. The code utilizes in large parts vectorization, and therefore, the running time is comparable to $\mathrm{C} / \mathrm{C}++$ implementations (see $[29,10]$ and [13] for a review and earlier references). FEEQS.M is publicly available and a forthcoming release will contain the here introduced coupling methods for plasma equilibrium calculations.

\subsection{Convergence}

We solve a simple magnetostatic problem in axial symmetry, which corresponds to a constant current carrying coil with poloidal section $\mathcal{C}_{1}=[0.5,1.5] \times$ $[-1.5,-0.5]$ :

$$
\begin{aligned}
-\nabla \cdot\left(\frac{1}{\mu_{0} x_{r}} \nabla \psi\right) & =\left\{\begin{array}{ll}
1 & \text { in } \mathcal{C}_{1} \\
0 & \text { elsewhere }
\end{array},\right. \\
\psi(\mathbf{x})_{\mid x_{r}=0} & =0 ; \quad \lim _{\|\mathbf{x}\| \rightarrow+\infty} \psi(\mathbf{x})=0 .
\end{aligned}
$$

With this simple linear test problem we can easily assess numerically the approximation quality of the four different approaches. The solution $\psi(\mathbf{x}$ ( of (26) and its gradient $\nabla \psi(\mathbf{x})$ for $\mathbf{x} \in \mathbb{H} \backslash \mathcal{C}_{1}$ are give as

$$
\psi(\mathbf{x})=\int_{\mathcal{C}_{1}} G(\mathbf{x}, \mathbf{y}) d \mathbf{y}, \quad \nabla \psi(\mathbf{x})=\int_{\mathcal{C}_{1}} \nabla_{\mathbf{x}} G(\mathbf{x}, \mathbf{y}) d \mathbf{y} .
$$

To study the convergence behavior of the different coupling approaches we introduce a second square $\mathcal{L}=[1,2] \times[0.5,1.5]$. The approaches HL, JN and BMC for solving (26) are based either on the computational domain $\Omega=\mathcal{L}$ or on the computational domain $\Omega=\mathcal{C}_{1} \cup \mathcal{L}$ for the finite element discretization. The first choice corresponds to the case when no source terms are in the computational domain $\Omega$, while the second choice is more relevant for the equilibrium problem, as it corresponds to the case when source terms, such as the plasma are in the computational domain $\Omega$. For ABB we choose $\Omega$ to be the half circle of radius 3 centered at $(0,0)$ that contains both $\mathcal{L}$ and $\mathcal{C}_{1}$ (see Figure 2 of an illustration). 

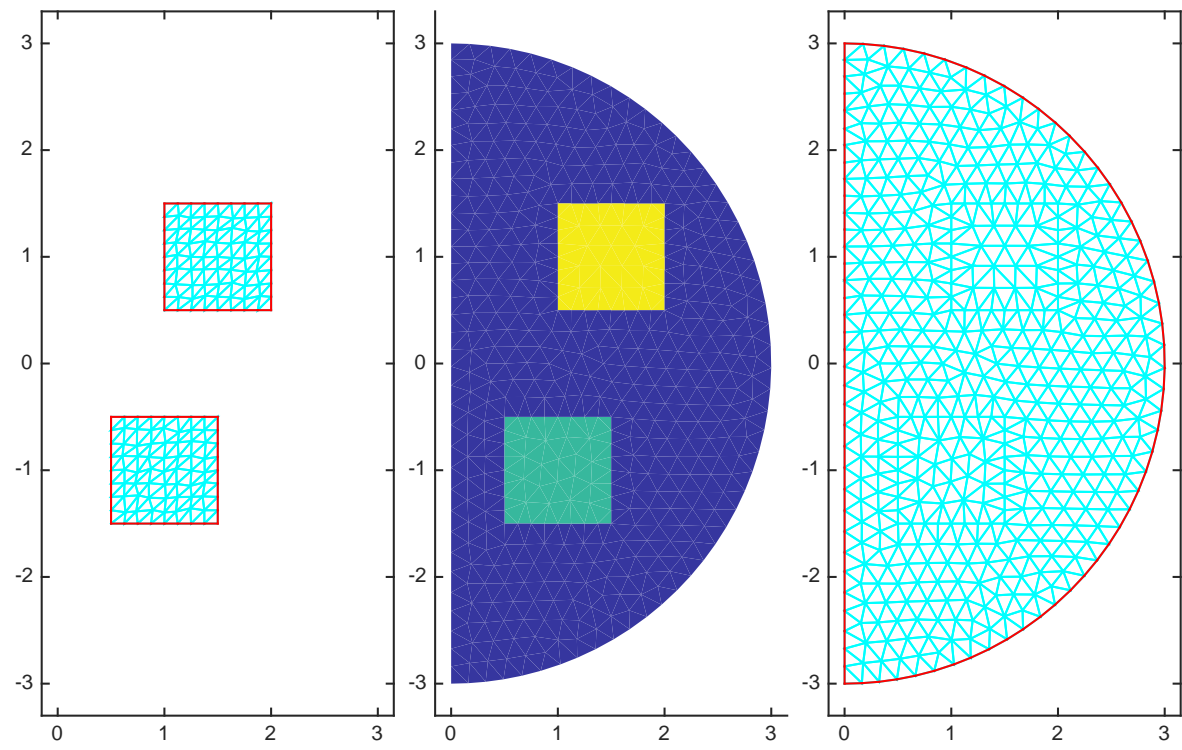

Figure 2: Center: The domain $\mathcal{C}_{1}$ (green) and the domain $L$ (yellow). Left: Example of the meshes used in the coupling methods HL, JN and BMC. Right: Example of the meshes used for the coupling method ABB.

As we consider here the linear problem the term $j_{p}(\psi, \cdot)$ vanishes in all four Galerkin formulations (20), (21), (22) and (23). Moreover, in the case of no sources in the computational domain, $\Omega=\mathcal{L}$, we have that $\ell(\cdot)$ vanishes while in the case of $\Omega=\mathcal{C}_{1} \cup \mathcal{L}$ both $L(\mathbf{x})$ and $L^{\prime}(\mathbf{x})$ vanish.

Then we compute the numerical solutions $\psi_{h}^{\mathrm{HL}}, \psi_{h}^{\mathrm{JN}}, \psi_{h}^{\mathrm{BMC}}$ and $\psi_{h}^{\mathrm{ABB}}$ with either of the four methods on a sequence of refined meshes and monitor the error in the domain $\mathcal{L}$ measured in the $L^{2}$-norm and the $H^{1}$-semi-norm:

$$
e r r_{0}^{M}=\sqrt{\int_{\mathcal{L}}\left(\psi_{h}^{\mathrm{M}}(\mathbf{x})-\psi(\mathbf{x})\right)^{2} d \mathbf{x}}, \quad \operatorname{err}_{1}^{M}=\sqrt{\int_{\mathcal{L}}\left|\nabla \psi_{h}^{\mathrm{M}}(\mathbf{x})-\nabla \psi(\mathbf{x})\right|^{2} d \mathbf{x}},
$$

where $\mathrm{M}$ runs through $\mathrm{JN}, \mathrm{HL}, \mathrm{BMC}$ and $\mathrm{ABB}$ and we use high precision quadrature for the convolution formulas in (27) to approximate $\psi(\mathbf{x})$ and $\nabla \psi(\mathbf{x})$. The results are shown in Figures 3 and 4.

First (see Figure 3, left), we look at the case when there are no sources in the computational domain. The numerical experiments confirm theoretical convergence assertions $[28,11]$ for the coupling methods JN and BMC: as we are using piecewise affine finite elements we observe second and first order convergence for $\mathrm{JN}$ in the $L^{2}$-norm and the $H^{1}$ semi-norm respectively. We are loosing one order of convergence for BMC in $L^{2}$, which is due to a loss of regularity of the auxiliary variable $q$ due to the corners of $\Omega$. This is a known [34, p. 149] disadvantage of indirect boundary integral methods such as BMC and we therefore exclude BMC from the subsequent discussion. 

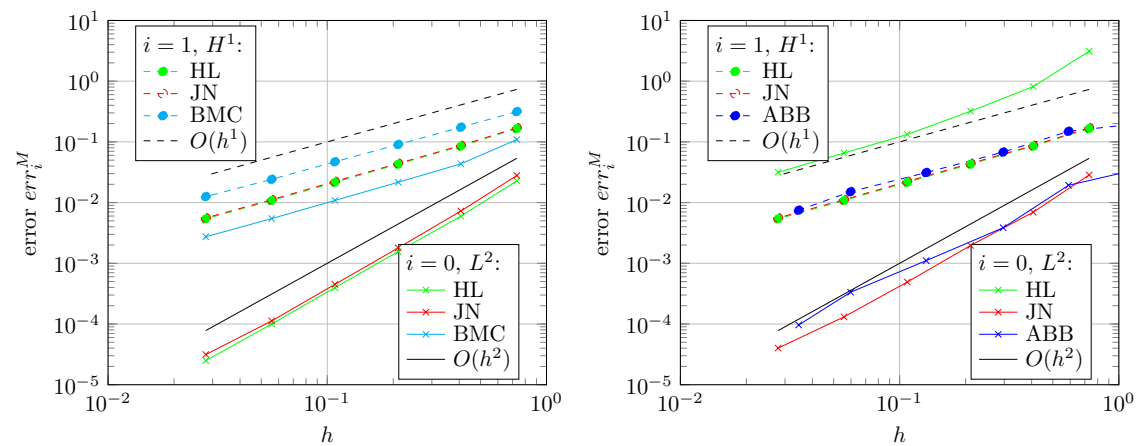

Figure 3: Left: Without sources in the computational domain, $\Omega=\mathcal{L}$ (not possible for ABB). Right: With sources in the computational domain, $\Omega=\mathcal{C}_{1} \cup \mathcal{L}$ for HL and JN and $\Omega^{\top}$ a half circle for $\mathrm{ABB}$.
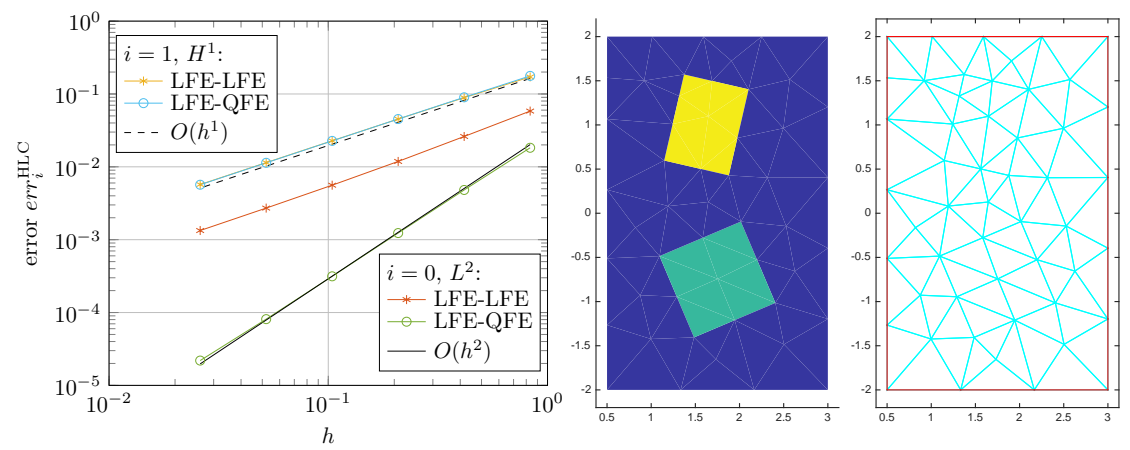

Figure 4: Left: The suboptimal convergence rate for $\psi^{\mathrm{HL}}$ in $L^{2}$ can be improved if we use quadratic finite elements (LFE-QFE) instead the linear finite elements (LFE-LFE) in (20) for the auxiliary variable $u$. Right: The computational domain $\Omega$ and the coarsest mesh, with the subdomains $\mathcal{L}$ (yellow), the domain where we evaluate the error and the domain $\mathcal{C}_{1}$ (green) the support of the source term. 

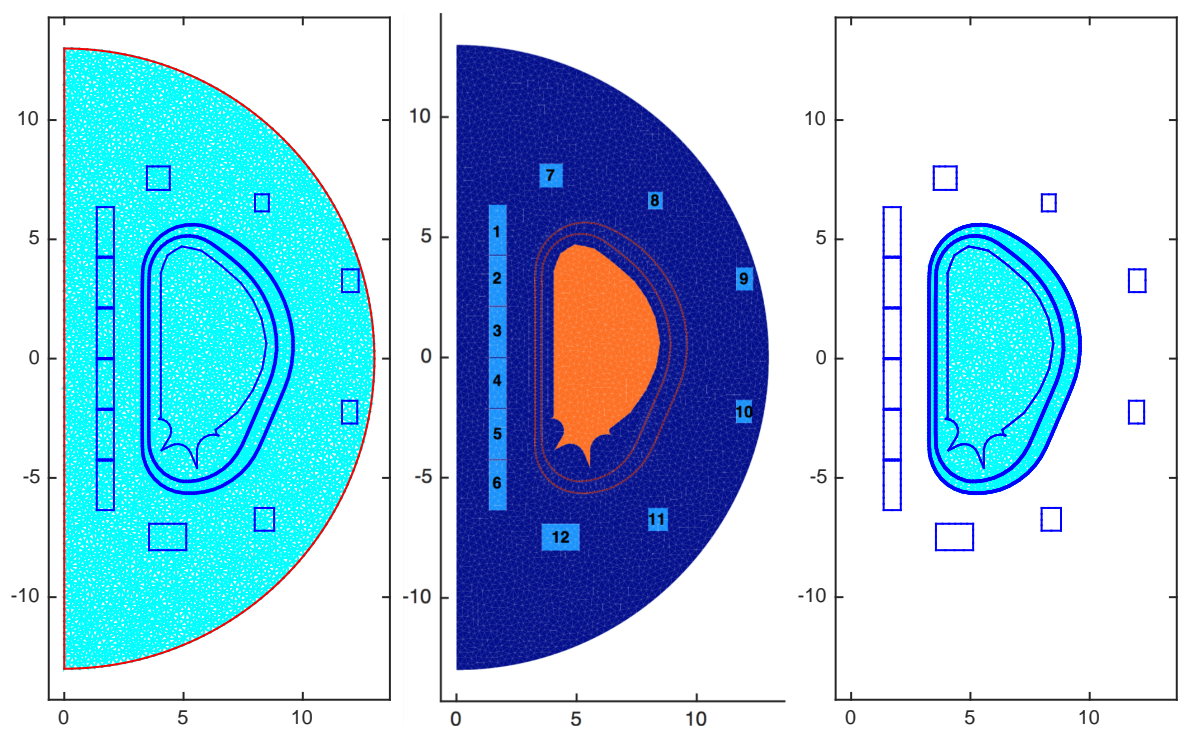

Figure 5: The ITER geometry (center) and the mesh for the domain $\Omega^{\mathbf{O}}$ (left) and the domain $\Omega$ (right). The coils are not included in $\Omega$.

To our knowledge there is no theoretical convergence analysis available for HL. While we see (see Figure 3, left) with $\Omega=\mathcal{L}$ as well first order convergence in the $H^{1}$-semi-norm, and second order convergence in the $L^{2}$-norm, we observe a loss of convergence for the case that sources are in the computational domain (see Figure 3, right). This is inherent to the method and a severe disadvantage of HL. A closer inspection of the last line of (20) shows, that HL replaces the missing Dirichlet data for $\psi$ by a convolution term with the Neumann data of the auxiliary variable $u$. Hence, the consistency error of this approximated Dirichlet data is of the same order as the consistency error for gradients of $u$ and $\psi$ and one order lower than the consistency error for $u$ and $\psi$. By a refined version of standard duality techniques in numerical analysis it can be shown that in Dirichlet problems with approximated Dirichlet data such a deteriorated consistency error leads to the non-optimal convergence, that we observe in our experiment [15, p. 91-93]. To cure this defect we would have to discretize the auxiliary variable $u$ with at least quadratic finite elements (see Figure 4), which then leads to an increase in the number of unknowns.

In the relevant case of sources in the computational domain, we observe a very similar convergence behavior of $\mathrm{ABB}$ and JN (see Figure 3, left).

In the following subsection we monitor the characteristic running times for each of the three approaches for a realistic equilibrium problem.

\subsection{Running time}

In the following we consider an example for ITER geometry (see Figure 5, center) with the coil currents indicated in the table in Figure 6. The current 

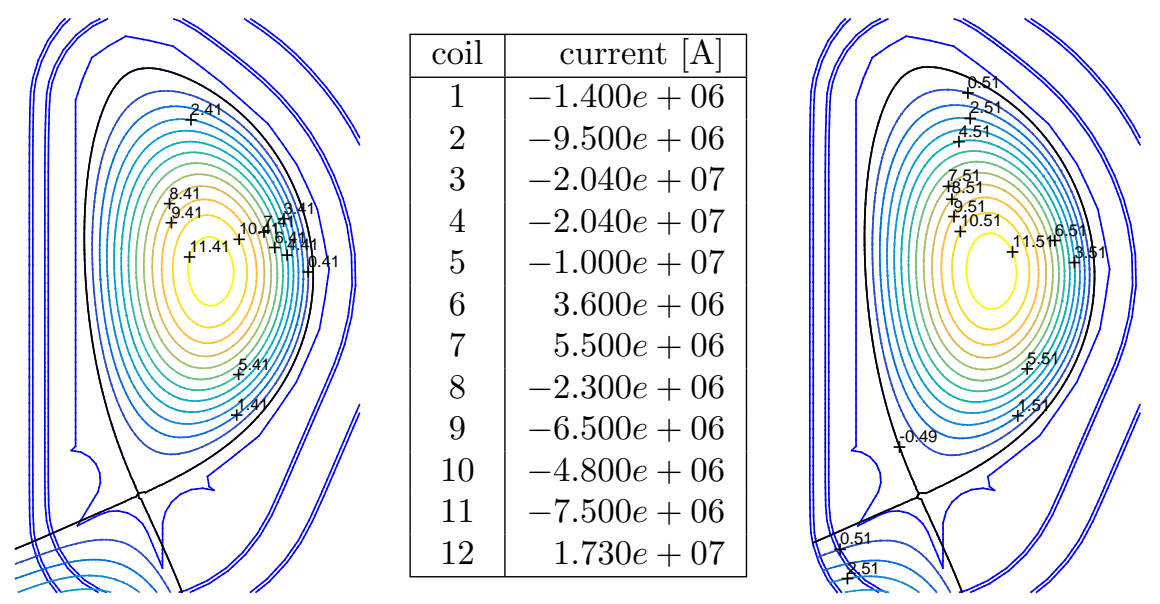

Figure 6: Case A: The currents in the coils (center) and contour plots of numerical solutions using ABB (left) and HL (right).

profile is the parametric profile

$$
j\left(x_{r}, \psi(\mathbf{x})\right)=\lambda\left(\beta \frac{x_{r}}{r_{0}}+(1-\beta) \frac{r_{0}}{x_{r}}\right)\left(1-\psi_{\mathrm{N}}(\mathbf{x})^{\alpha}\right)^{\gamma}
$$

with $r_{0}=6.2 \mathrm{~m}$ the major radius of the vacuum chamber and $\alpha=2.0, \beta=$ $0.5978, \gamma=1.395$ and $\lambda=1.365461 e+6 . \psi_{\mathrm{N}}$ the normalized poloidal flux

$$
\psi_{\mathrm{N}}(\mathbf{x})=\frac{\psi(\mathbf{x})-\psi_{\mathrm{ax}}(\psi)}{\psi_{\mathrm{bd}}(\psi)-\psi_{\mathrm{ax}}(\psi)}
$$

where $\psi_{\mathrm{ax}}$ and $\psi_{\mathrm{bd}}$ are the flux values at the magnetic axis and the plasma boundary $\partial \mathcal{P}(\psi)$. Exemplary meshes for ABB and HL/JN are shown in Figure 5. HL and JN are based on a mesh that covers the domain bounded by the outer vacuum vessel wall. The initial guesses are solutions to equilibrium problems with fixed, prescribed plasma current, and then the Newton iterations converge to a residual smaller then $10^{-12}$ in less then 10 iterations. The difference between the numerical solutions of ABB, HL and JN is negligible (see Figure 6 left and right), so we can focus on the runtime. As all the three methods are implemented in the same environment, this is a fair test to assess the performance of each approach. A more sophisticated implementation that allows to improve the performance of one method, will also improve the performance of the two other methods.

The pseudo-code for Newton-type schemes can be found in Figure 7. In our first test, we look at the timing of the pre-processing, line 1 in the pseudo-code, and time per Newton iteration, the update step in lines 3 and 4 in the pseudocode (see Figure 7). The pre-processing steps consists mainly of the assembling of all stiffness matrices that do not change during the Newton iterations. This involves in particular the assembling of all boundary integral terms, that has in general quadratic complexity due to the convolution terms. The main effort in 

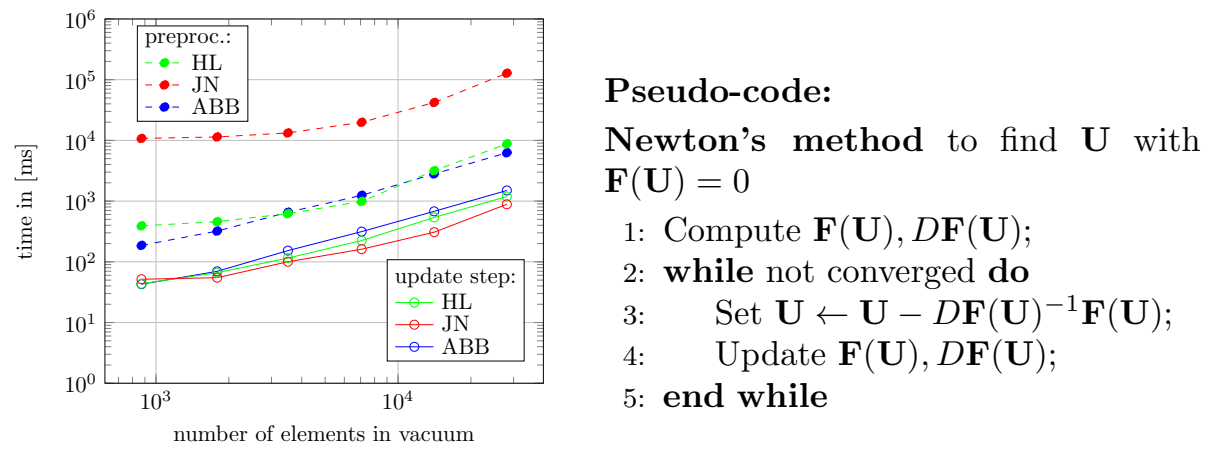

Figure 7: Timing of the pre-processing (preproc.), i.e. line 1 in Newton's method, and time per Newton Iteration (update step), i.e. lines 3 and 4 in Newton's method, for the different coupling methods.

the update step is due to the inversion of the Newton matrix and the update of the plasma domain and its corresponding terms, i.e. $j_{p}(\psi, \xi)$ in the Galerkin formulations. We show in Figure 7 characteristic timings of the pre-processing step and the update step as functions of the number triangles that cover the domain accessible by the plasma (orange in Figure 5), as this number is identical for both type of meshes. Saying this, it is obvious that the pre-processing time for $\mathrm{JN}$ is the largest, as it contains more boundary integral terms with convolutions than ABB and HL. It is a bit surprising that there is not a huge difference in the timing of the update step itself, even though the total number of unknowns for JN, ABB and HL are quite different (see Table 2). The total number of unknowns of HL is roughly twice as large as the total number of unknowns of JN, which is also obvious from the Galerkin formulations (20) and (21). And the number of unknowns of ABB is considerably larger than the number of unknowns of HL. Updating the plasma domain and the corresponding terms (line 4 in the pseudo-code) is very similar in all three methods. A closer inspection of the timings of lines 3 and 4 in the pseudo-code (see column 4-9 in Table 2) uncovers that the inversion of the Newton matrix $D \mathbf{F}(\mathbf{U})$ is the most time consuming part of the update steps. Moreover the timing of the solution step for HL and ABB is comparable to the timing for JN even though the number of unknowns are much larger. After all this is not very surprising, if one looks at the structure, e.g. the sparsity pattern (see Figure 8), of the different Newton matrices. Due to the integral equations the matrices for HL and JN contain relatively large dense blocks, while ABB overall remains a sparse matrix. This difference explains the observed timings. It might be possible to design problem adapted linear solvers that speed up the inversion of the Newton matrix for HL or JN, but as we are relying here on high-performance software (MATLAB's proprietary interface to UMFPACK [14]), it will be difficult to do better. 

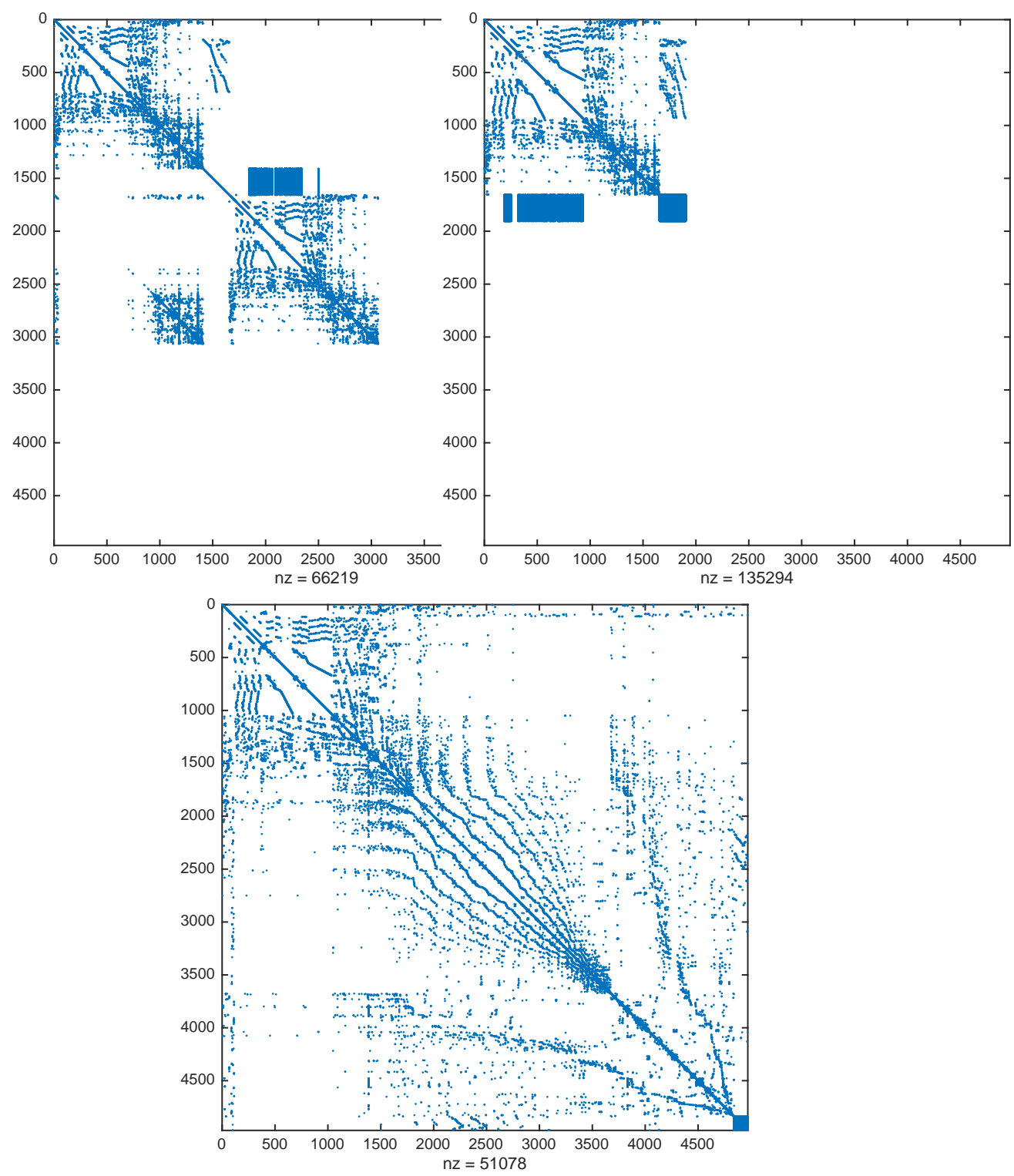

Figure 8: The sparsity pattern for the Newton matrix $D \mathbf{F}$ (see Fig 7 ) for HL, JN and ABB (from left to right, top to down). The matrix $D \mathbf{F}$ for $\mathrm{ABB}$ is the largest but has the least number of nonzero entries. 


\begin{tabular}{|c|c|c||c|c|c||c|c|c|}
\hline \multicolumn{3}{|c||}{ number of unknowns } & \multicolumn{3}{c||}{ time per iteration $[\mathrm{ms}]$} & \multicolumn{4}{|c|}{ time per solve $[\mathrm{ms}]$} \\
\hline $\mathrm{HL}$ & $\mathrm{JN}$ & $\mathrm{ABB}$ & $\mathrm{HL}$ & $\mathrm{JN}$ & $\mathrm{ABB}$ & $\mathrm{HL}$ & $\mathrm{JN}$ & $\mathrm{ABB}$ \\
\hline $3.1 e+3$ & $1.9 e+3$ & $5.0 e+3$ & $4.4 e+1$ & $5.2 e+1$ & $4.3 e+1$ & $3.4 e+1$ & $4.2 e+1$ & $2.8 e+1$ \\
$4.5 e+3$ & $2.6 e+3$ & $8.8 e+3$ & $6.6 e+1$ & $5.5 e+1$ & $7.0 e+1$ & $5.1 e+1$ & $4.4 e+1$ & $4.6 e+1$ \\
$7.7 e+3$ & $4.2 e+3$ & $1.7 e+4$ & $1.2 e+2$ & $1.0 e+2$ & $1.5 e+2$ & $8.8 e+1$ & $7.5 e+1$ & $1.1 e+2$ \\
$1.5 e+4$ & $7.7 e+3$ & $3.3 e+4$ & $2.2 e+2$ & $1.6 e+2$ & $3.1 e+2$ & $1.8 e+2$ & $1.2 e+2$ & $2.3 e+2$ \\
$2.9 e+4$ & $1.5 e+4$ & $6.6 e+4$ & $5.4 e+2$ & $3.1 e+2$ & $6.8 e+2$ & $4.4 e+2$ & $2.4 e+2$ & $5.0 e+2$ \\
$5.7 e+4$ & $2.9 e+4$ & $1.3 e+5$ & $1.2 e+3$ & $8.9 e+2$ & $1.5 e+3$ & $1.0 e+3$ & $7.6 e+2$ & $1.1 e+3$ \\
\hline
\end{tabular}

Table 2: Timing results for the coupling methods HL, JN and ABB. One "iteration" corresponds to line 3 and 4 from Newton's method in Fig. 7, whereas "solve" corresponds to line 3 alone.

\subsection{Fixed point vs Newton Iteration}

It is well known $[30,27]$ that plain fixed point iterations for solving the non-linear Galerkin formulations (20), (21), (22) and (23) suffer from sever convergence problems. It is also known, but far less widespread, that Newtontype methods avoid such convergence problems. In [6, Section IV 1.5.1] for example it was shown, in the simplified setting of the TFR tokamak, that one can find solutions of the equilibrium problem using Newton type methods, that can not be found with fixed point iterations. The subsequent numerical experiments underpin this observation.

Additionally to the equilibrium from the previous section (case A, see Figure 6) we consider two equilibria with circular boundary that have a contact point with the left (case B), respectively the right (case C) side of the limiter (see Figures 9 and 10). We used an inverse problem formulation with prescribed desired boundary [20, Section 2.2] to identify currents (see the tables in Figures 9 and 10). Again as in the case A, the Newton methods for ABB and HL converge also for the case $\mathrm{B}$ and $\mathrm{C}$ in less than 10 iterations, where here we took for simplicity random perturbations of the numerical solution as initial guess. As we do not focus on global convergence this is reasonable. But it is important to understand the behavior of fixed point iterations for such random perturbation. In figure 11 we present the convergence history of fixed-point iterations for $\mathrm{ABB}$ and $\mathrm{HL}$ for the three different test cases. We observe that the fixed point iterations for $\mathrm{ABB}$ and $\mathrm{HL}$ do not converge for the test cases $\mathrm{A}$ and $\mathrm{B}$ and that the convergence for the test case $\mathrm{C}$ is extremely slow. Fixed point iterations can fail both for elongated as well as circular equilibria. To show that this observation it not related to our choice of perturbation, we recall that the convergence of fixed point iterations is determined by the spectral radius $\rho_{D \mathbf{G}(\mathbf{U})}$ (maximum among the absolute values of the eigenvalues of $D \mathbf{G}(\mathbf{U})$ ), where $D \mathbf{G}(\mathbf{U})$ is the Jacobian of the function $\mathbf{G}(\mathbf{U})$ that defines the fixed point iteration:

$$
\mathbf{U}^{k+1}=\mathbf{G}\left(\mathbf{U}^{k}\right) .
$$

We have convergence of the sequence $\mathbf{U}^{k}$ to the fixed point $\mathbf{U}^{*}$, with $\mathbf{U}^{*}=$ 


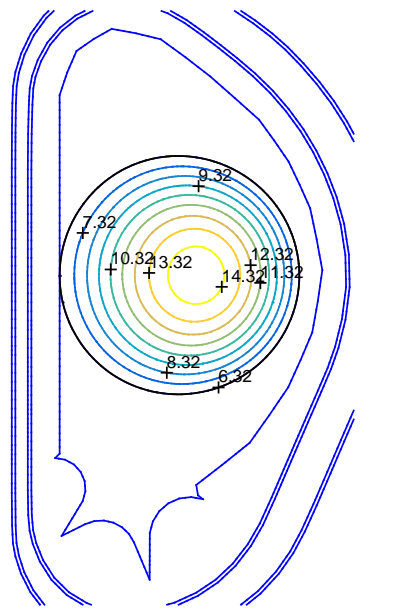

\begin{tabular}{|c|r|}
\hline coil & current $[\mathrm{A}]$ \\
\hline 1 & $-8.208 e+05$ \\
2 & $-8.621 e+04$ \\
3 & $2.783 e+06$ \\
4 & $1.703 e+06$ \\
5 & $-6.491 e+05$ \\
6 & $-7.971 e+05$ \\
7 & $-2.026 e+06$ \\
8 & $-2.508 e+06$ \\
9 & $-2.150 e+06$ \\
10 & $-1.874 e+06$ \\
11 & $-3.607 e+06$ \\
12 & $-2.303 e+06$ \\
\hline
\end{tabular}

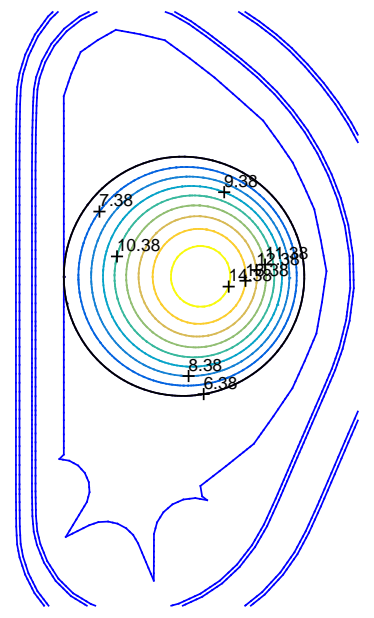

Figure 9: Case B: The currents in the coils (center) and contour plots of numerical solutions using $\mathrm{ABB}$ (left) and HL (right).

$\mathbf{G}\left(\mathbf{U}^{*}\right)$ if the spectral radius $\rho_{D \mathbf{G}\left(\mathbf{U}^{*}\right)}$ is smaller than one. Since we are able to compute the derivatives required for Newton-type iterations, we are also able to compute the derivatives of the functions $\mathbf{G}$ that define the fixed point iterations for ABB and HL. The power iteration method in turn allows to compute the spectral radius. Computing the spectral radius, the convergence indicator, for the example from Figure 11, we find that indeed its value is larger than one in the cases where we don't observe convergence (see legend of Figure 11 for the numbers). Moreover, in case $\mathrm{C}$ where we see convergence, the spectral radius is smaller than one. Nevertheless, its values are still fairly large, which explains the extremely slow speed of convergence.

Ultimately, we would like to stress that the size of the spectral radius, hence the success of fixed point iterations is not related to the discretization parameter. In table 3 we show the values of the spectral radius of fixed point iterations (29) for $\mathrm{ABB}$ and $\mathrm{HL}$ for the three different test cases for sequence of finer and finer meshes. Newton's method converges to the same equilibrium as indicated by the numbers in the columns with header vol. giving the total plasma volume, but the values of the spectral radius remain almost constant.

\section{Conclusion}

We presented a systematic discussion of four different approaches to the approximation of free-boundary equilibrium problems which are consistent with the boundary condition at infinity. All four methods utilize boundary integral equations. HL, the most common method for such kind of applications, uses a boundary integral equation to derive non-local Dirichlet conditions on the boundary of the computational domain, while the other three approaches are rather based on non-local Neumann conditions. ABB, introduced in [2], requires 


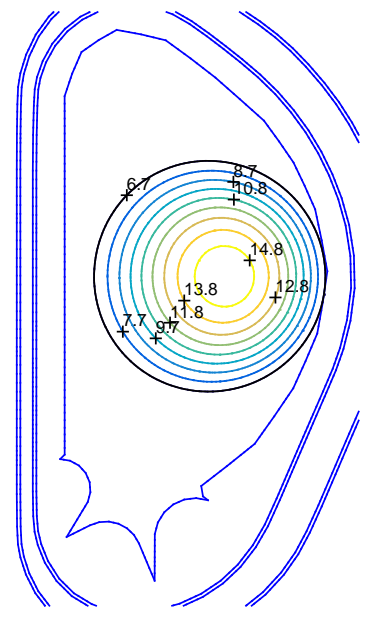

\begin{tabular}{|c|r|}
\hline coil & current $[\mathrm{A}]$ \\
\hline 1 & $-6.705 e+05$ \\
2 & $1.373 e+04$ \\
3 & $2.133 e+06$ \\
4 & $1.432 e+06$ \\
5 & $-3.774 e+05$ \\
6 & $-6.172 e+05$ \\
7 & $-1.885 e+06$ \\
8 & $-2.359 e+06$ \\
9 & $-2.124 e+06$ \\
10 & $-1.836 e+06$ \\
11 & $-3.491 e+06$ \\
12 & $-2.040 e+06$ \\
\hline
\end{tabular}

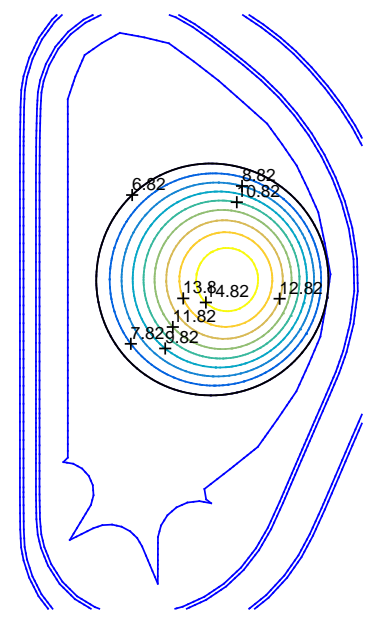

Figure 10: Case C: The currents in the coils (center) and contour plots of numerical solutions using ABB (left) and HL (right).

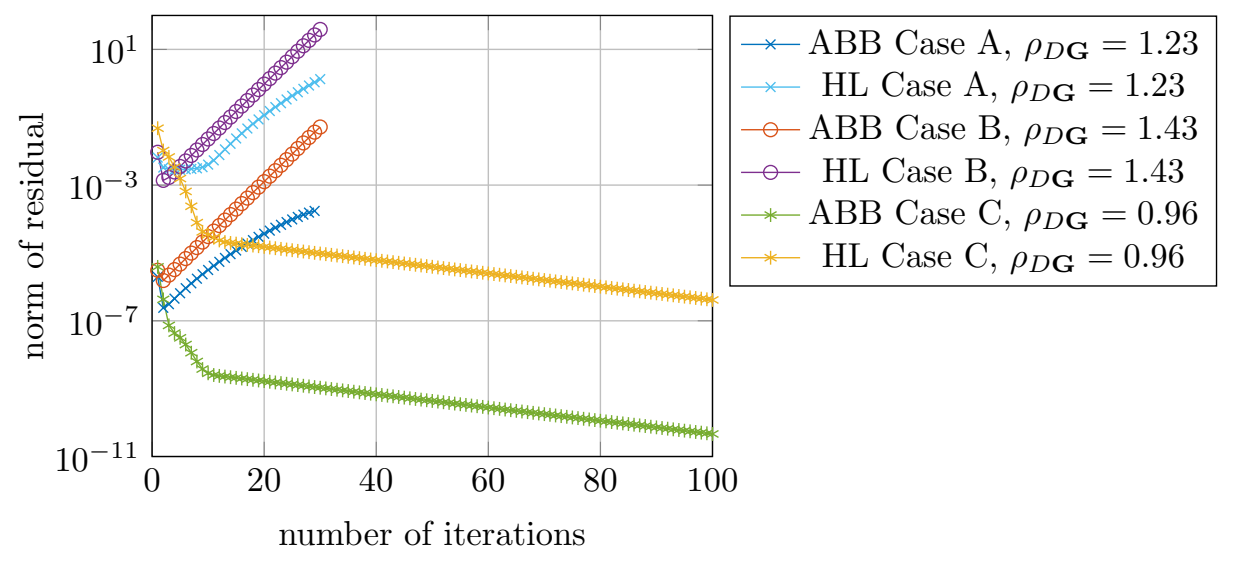

Figure 11: Convergence history of fixed point iterations for ABB and HL for the three different test cases A, B and C. 


\begin{tabular}{|c|c|c|c|c|c|c|c|c|c|}
\hline & \multicolumn{3}{|c|}{ Case A } & \multicolumn{3}{|c|}{ Case B } & \multicolumn{3}{|c|}{ Case C } \\
\hline \multirow[t]{2}{*}{$\mathrm{h}$} & vol. $\left[m^{3}\right]$ & \multicolumn{2}{|c|}{$\rho_{D \mathbf{G}}$} & vol. $\left[m^{3}\right]$ & \multicolumn{2}{|c|}{$\rho_{D \mathbf{G}}$} & vol. $\left[m^{3}\right]$ & \multicolumn{2}{|c|}{$\rho_{D \mathbf{G}}$} \\
\hline & & $\mathrm{ABB}$ & HL & & $\mathrm{ABB}$ & HL & & $\mathrm{ABB}$ & HL \\
\hline 0.22 & 845.13 & 1.29 & 1.25 & 490.88 & 1.47 & 1.47 & 489.84 & 0.95 & 0.95 \\
\hline 0.16 & 834.72 & 1.24 & 1.24 & 486.81 & 1.46 & 1.45 & 486.34 & 0.96 & 0.96 \\
\hline 0.11 & 832.26 & 1.23 & 1.23 & 484.56 & 1.43 & 1.43 & 486.22 & 0.96 & 0.96 \\
\hline 0.08 & 831.04 & 1.25 & 1.24 & 484.88 & 1.45 & 1.45 & 485.75 & 0.96 & 0.96 \\
\hline 0.06 & 830.24 & 1.25 & 1.24 & 484.62 & 1.45 & 1.46 & 485.41 & 0.96 & 0.96 \\
\hline 0.04 & 830.52 & 1.24 & 1.24 & 484.38 & 1.45 & 1.46 & 485.07 & 0.96 & 0.96 \\
\hline
\end{tabular}

Table 3: The spectral radius of fixed point iterations for ABB and HL on a sequence of refined meshes.

the computational domain to be a semi-circle, which can lead to a relatively large number of unknowns. The two standard methods JN and BMC were never used before in free-boundary equilibrium problems.

We have shown, for the first time, that HL suffers from non-optimal convergence, compared to $\mathrm{ABB}$ and JN. This problem can be cured (see Figure 4) using higher order approximations for the auxiliary variable, but only at the cost of increased computational complexity. We do not recommend BMC for equilibrium calculations as this approach will be non-optimal in computational domains with corners [34, p. 149]. ABB is used in a family of equilibrium codes such as Proteus [3], CREATE-NL+ [1] or CEDRES++ and FEEQS.M [19, 20], but the relatively large computational domain is often considered to be a main drawback. We have shown, that nevertheless the method can be competitive, as it avoids integral equations that lead to dense linear algebraic systems. Our numerical examples show that the computation time of ABB is comparable to HL or JN even though the number of degrees of freedom is much larger. If one prefers to stay with smaller computational domains, then JN is a better choice than HL, but has not yet appeared in equilibrium codes. For a very long time HL was promoted for its combination of two Dirichlet problems, that could be solved efficiently with standard iterative solvers. Today's powerful direct solvers make this argument obsolete for many applications.

Moreover, our experiments give again evidence that it is inevitable to use Newton-type iterations in order to solve the non-linear discrete problems. The observation is similar to the one in [6] for the case of radial instabilities. Knowing that most of today's equilibrium codes follow the spirit and ideas of von Hagenow and Lackner [39, 30], and employ some sort of HL combined with fixed point iterations, it is important to make aware the limits of such an approach. We strongly advocate Newton type methods to circumvent the convergence problems for physically unstable equilibria. Augmenting an existing code based on a fixed-point solver with a Newton-type solver is, at first glance, fairly technical. But then, a closer look, shows that this is only slightly more complicated than the computation of the plasma domain itself and details can be found in the 
existing literature [20].

\section{Acknowledgements}

The authors thank the referees for their comments and suggestions. These led to a significantly improved revised version. We thank O. de Barbieri for the dicussions on $\mathrm{ABB}$ and the copy of the reference [2].

[1] R. Albanese, R. Ambrosino, and M. Mattei. CREATE-NL+: A robust control-oriented free boundary dynamic plasma equilibrium solver. Fusion Engineering and Design, 9697:664 - 667, 2015. Proceedings of the 28th Symposium On Fusion Technology (SOFT-28).

[2] R. Albanese, J. Blum, and O. De Barbieri. On the solution of the magnetic flux equation in an infinite domain. In EPS. 8th Europhysics Conference on Computing in Plasma Physics (1986), pages 41-44, 1986.

[3] R. Albanese, J. Blum, and O. De Barbieri. Numerical studies of the Next European Torus via the PROTEUS code. In 12th Conf. on Numerical Simulation of Plasmas, San Francisco, 1987.

[4] A. Bermudez, D. Gomez, M. C. Muniz, and P. Salgado. A FEM/BEM for axisymmetric electromagnetic and thermal modelling of induction furnaces. International Journal for Numerical Methods in Engineering, 71(7):856878, 2007.

[5] J. Bielak and R. C. MacCamy. An exterior interface problem in twodimensional elastodynamics. Quart. Appl. Math., 41(1):143-159, 1983/84.

[6] J. Blum. Numerical simulation and optimal control in plasma physics. Wiley/Gauthier-Villars, 1989.

[7] J. Blum, C. Boulbe, and B. Faugeras. Real-time plasma equilibrium reconstruction in a tokamak. In Journal of Physics: Conference Series. Proceedings of the 6th International Conference on Inverse Problems in Engineering: Theory and Practice, volume 135, page 012019. IOP Publishing, 2008.

[8] J. Blum, C. Boulbe, and B. Faugeras. Reconstruction of the equilibrium of the plasma in a tokamak and identification of the current density profile in real time. Journal of Computational Physics, 231(3):960 - 980, 2012.

[9] J. Blum, J. Le Foll, and B. Thooris. The self-consistent equilibrium and diffusion code SCED. Computer Physics Communications, 24:235 - 254, 1981.

[10] L. Chen. Programming of finite element methods in Matlab, 2011. 
[11] M. Costabel, V.J. Ervin, and E.P. Stephan. Experimental convergence rates for various couplings of boundary and finite elements. Mathematical and Computer Modelling, 15(3):93 - 102, 1991.

[12] M. Costabel and E.P. Stephan. Coupling of finite and boundary element methods for an elastoplastic interface problem. SIAM J. Numer. Anal., 27(5):1212-1226, 1990.

[13] F. Cuvelier, C. Japhet, and G. Scarella. An efficient way to assemble finite element matrices in vector languages. BIT, 56(3):833-864, 2016.

[14] T.A. Davis. Umfpack http://www.cise.ufl.edu/research/sparse/ umfpack/, 2011.

[15] G. Fairweather. Finite element Galerkin methods for differential equations. Marcel Dekker, Inc., New York-Basel, 1978. Lecture Notes in Pure and Applied Mathematics, Vol. 34.

[16] J. P. Freidberg. Ideal Magnetohydrodynamics. Plenum US, 1987.

[17] G.N. Gatica and G.C. Hsiao. The uncoupling of boundary integral and finite element methods for nonlinear boundary value problems. J. Math. Anal. Appl., 189(2):442-461, 1995.

[18] J. P. Goedbloed and S. Poedts. Principles of magnetohydrodynamics: with applications to laboratory and astrophysical plasmas. Cambridge university press, 2004.

[19] V. Grandgirard. Modélisation de l'équilibre d'un plasma de tokamak. PhD thesis, Université de Franche-Comté, 1999.

[20] H. Heumann, J. Blum, C. Boulbe, B. Faugeras, G. Selig, J.-M. Ané, S. Brémond, V. Grangirard, P. Hertout, and E. Nardon. Quasi-static freeboundary equilibrium of toroidal plasma with CEDRES++: computational methods and applications. J. Plasma Physics, 2015.

[21] F.L. Hinton and R.D. Hazeltine. Theory of plasma transport in toroidal confinement systems. Rev. Mod. Phys., 48:239-308, Apr 1976.

[22] R. Hiptmair. Coupling of finite elements and boundary elements in electromagnetic scattering. SIAM J. Numer. Anal., 41(3):919-944, 2003.

[23] G. C. Hsiao and S. Zhang. Optimal order multigrid methods for solving exterior boundary value problems. SIAM J. Numer. Anal., 31(3):680-694, 1994.

[24] M. Itagaki and T. Fukunaga. Boundary element modelling to solve the Grad-Shafranov equation as an axisymmetric problem. Engineering Analysis with Boundary Elements, 30(9):746 - 757, 2006. 
[25] M. Itagaki, J. Kamisawada, and S. Oikawa. Boundary-only integral equation approach based on polynomial expansion of plasma current profile to solve the Grad-Shafranov equation. Nuclear Fusion, 44(3):427, 2004.

[26] J.D. Jackson. Classical electrodynamics. Wiley, 1975.

[27] S.C. Jardin. Computational methods in plasma physics. Boca Raton, FL : CRC Press/Taylor \& Francis, 2010.

[28] C. Johnson and J. C. Nedelec. On the coupling of boundary integral and finite element methods. Mathematics of Computation, 35(152):pp. 10631079, 1980.

[29] J. Koko. Vectorized Matlab codes for linear two-dimensional elasticity. Sci. Program., 15(3):157-172, August 2007.

[30] K. Lackner. Computation of ideal MHD equilibria. Computer Physics Communications, 12(1):33 - 44, 1976.

[31] L.L. Lao, J.R. Ferron, R.J. Geoebner, W. Howl, H.E. St. John, E.J. Strait, and T.S. Taylor. Equilibrium analysis of current profiles in Tokamaks. Nuclear Fusion, 30(6):1035, 1990.

[32] P.J. Mc Carthy, P. Martin, and W. Schneider. The CLISTE Interpretive Equilibrium Code. Technical Report IPP Report 5/85, Max-Planck-Institut fur Plasmaphysik, 1999.

[33] J.-M. Moret, B.P. Duval, H.B. Le, S. Coda, F. Felici, and H. Reimerdes. Tokamak equilibrium reconstruction code LIUQE and its real time implementation. Fusion Engineering and Design, 91(0):1-15, 2015.

[34] Stefan A. Sauter and Christoph Schwab. Boundary element methods, volume 39 of Springer Series in Computational Mathematics. Springer-Verlag, Berlin, 2011. Translated and expanded from the 2004 German original.

[35] F.-J. Sayas. The validity of Johnson-Nedelec BEM-FEM coupling on polygonal interfaces. SIAM Review, 55(1):131-146, 2013.

[36] O. Steinbach. Numerical approximation methods for elliptic boundary value problems. Springer, New York, 2008. Finite and boundary elements, Translated from the 2003 German original.

[37] E.P. Stephan. Coupling of finite elements and boundary elements for some nonlinear interface problems. Comput. Methods Appl. Mech. Engrg., 101(13):61-72, 1992.

[38] T. Takeda and S. Tokuda. Computation of MHD equilibrium of tokamak plasma. J. Comput. Phys., 93(1):1-107, 1991. 
[39] K. von Hagenow and K. Lackner. Computation of axisymmetric MHD equilibria. In 7th Conf. on Numerical Simulation of Plasmas, New York, page 140, 1975.

[40] J. Wesson. Tokamaks. The International Series of Monographs in Physics. Oxford University Press, 2004.

[41] K. Zhao, M.N. Vouvakis, and J.-F. Lee. Solving electromagnetic problems using a novel symmetric FEM-BEM approach. Magnetics, IEEE Transactions on, 42(4):583-586, April 2006.

[42] O.C Zienkiewicz, D.W. Kelly, and P. Bettess. Marriage à la mode - the best of both worlds (finite elements and boundary integrals). In R. Glowinski, E.Y. Rodin, and O.C. Zienkiewicz, editors, Energy Methods in Finite Element Analysis, pages 81-107. Wiley, Chichester, UK, 1979. 